\title{
Ensemble model output statistics for wind vectors
}

\author{
Nina Schuhen, Thordis L. Thorarinsdottir and Tilmann Gneiting \\ Institute of Applied Mathematics, University of Heidelberg, Germany
}

January 13,2012

\begin{abstract}
A bivariate ensemble model output statistics (EMOS) technique for the postprocessing of ensemble forecasts of two-dimensional wind vectors is proposed, where the postprocessed probabilistic forecast takes the form of a bivariate normal probability density function. The postprocessed means and variances of the wind vector components are linearly bias-corrected versions of the ensemble means and ensemble variances, respectively, and the conditional correlation between the wind components is represented by a trigonometric function of the ensemble mean wind direction. In a case study on 48-hour forecasts of wind vectors over the North American Pacific Northwest with the University of Washington Mesoscale Ensemble, the bivariate EMOS density forecasts were calibrated and sharp, and showed considerable improvement over the raw ensemble and reference forecasts, including ensemble copula coupling.
\end{abstract}

\section{Introduction}

The past two decades have seen a change of paradigms in weather forecasting, in that ensemble prediction systems have been developed and implemented operationally (Leutbecher and Palmer, 2008). Ensemble systems seek to reflect and quantify sources of uncertainty in numerical weather forecasts, such as imperfections in initial conditions and incomplete mathematical representations of the atmosphere. Despite the ubiquitous positive spread-skill relationship (Whitaker and Loughe, 1998; Grimit and Mass, 2002), ensemble forecasts tend to be biased, and typically they are underdispersed (Hamill and Colucci, 1997), in that the ensemble spread is too small to be realistic. Furthermore, differing spatial resolutions of the forecast grid and the observation network may need to be reconciled.

To address these shortcomings, various techniques for the statistical postprocessing of ensemble model output have been developed (Wilks and Hamill, 2007), with ensemble model output statistics (EMOS) or nonhomogeneous Gaussian regression (Gneiting et al., 2005; Thorarinsdottir and Gneiting, 2010) being a state of the art method. The EMOS technique transforms a raw ensemble forecast into a predictive probability density function, and simultaneously corrects for biases and dispersion errors. EMOS methods have been developed for temperature and surface pressure (Gneiting et al., 2005; Hagedorn et al., 2008; Kann et al., 2009), where the predictive density is normal and the method is often referred to as nonhomogeneous Gaussian regression, for quantitative precipitation (Wilks, 2009), and for wind speed (Thorarinsdottir and Gneiting, 2010; Thorarinsdottir and Johnson, 2011). In all these implementations, the predictive density applies to a univariate weather quantity. 


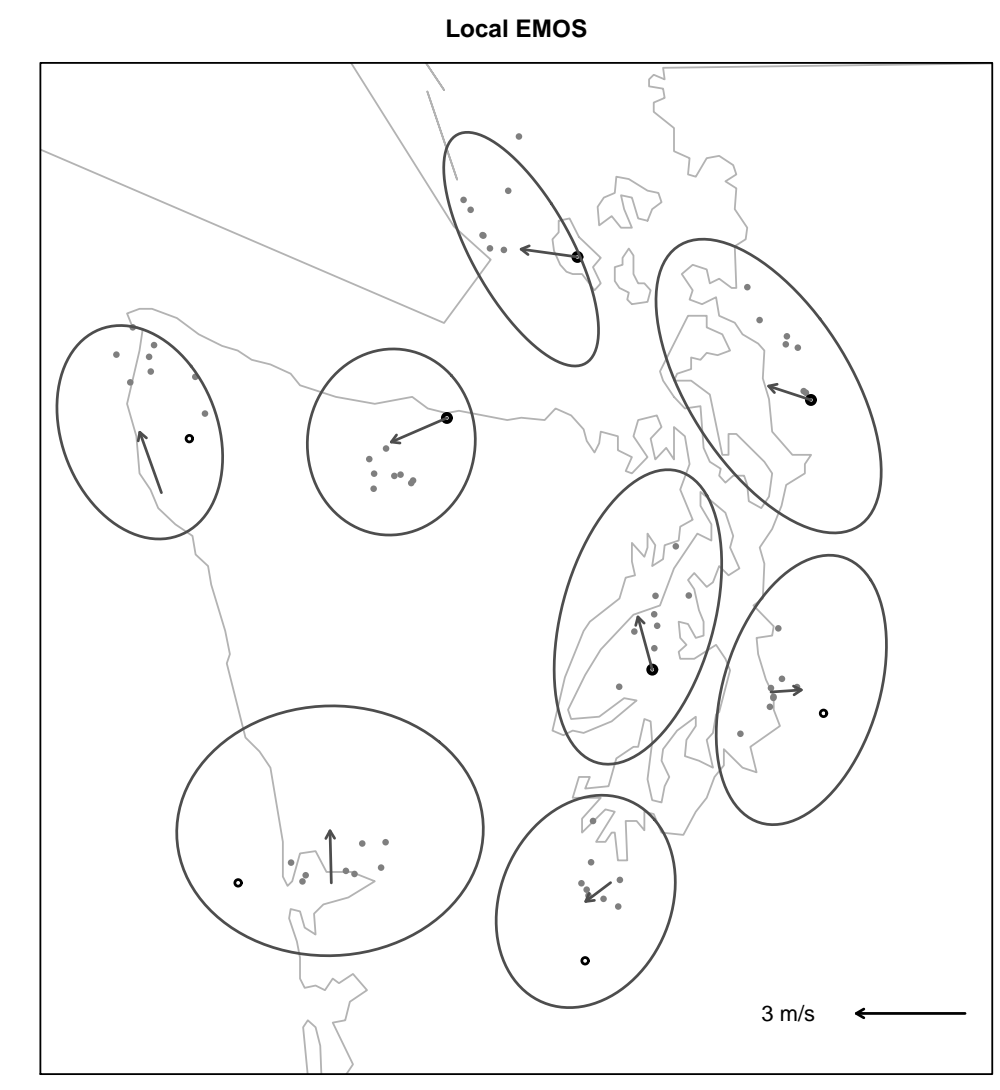

Figure 1: Raw ensemble and EMOS postprocessed forecasts of surface wind vectors at stations in the Olympic Peninsula and Puget Sound area in the US state of Washington, valid October 20, 2008 at 00 UTC, at a prediction horizon of 48 hours. The eight members of the University of Washington Mesoscale Ensemble (UWME; Eckel and Mass 2005) are shown as gray dots. The 75\% prediction ellipse and the mean vector for the EMOS density forecast are shown in dark gray, and the verifying wind vector is represented by a small black circle.

In this current paper, we propose and develop an EMOS technique for a bivariate weather quantity, namely surface wind vectors, comprising both zonal and meridional components or, in an alternative but mathematically equivalent representation, wind speed and wind direction. Probabilistic forecasts of wind conditions are critical in a wide range of applications, including air traffic control, ship routing, recreational and competitive sailing, and wind energy, where their societal and monetary value is huge (Marquis et al., 2011). Until very recently, wind speed and wind direction have been addressed independently in statistical postprocessing, without taking dependencies into account (Bao et al., 2010; Sloughter et al., 2010; Thorarinsdottir and Gneiting, 2010; Thorarinsdottir and Johnson, 2011). However, in many of the aforementioned applications it is important to honor the full information about the bivariate structure of the future wind vector that is provided by the ensemble. Thus, our EMOS postprocessed forecasts take the form of elliptically symmetric bivariate normal densities, as illustrated in Figure 1 in an application to the University of Washington Mesoscale Ensemble (UWME; Eckel and Mass 2005).

The remainder of the paper is organized as follows. In Section 2 we provide the details of 
the bivariate EMOS technique. A case study is presented in Section 3, where we consider 48hour ahead forecasts of wind vectors over the North American Pacific Northwest in 2008 based on the eight-member UWME. The paper closes in Section 4, where we hint at future developments and discuss the similarities and differences between our EMOS technique, the BMA approach of Sloughter (2009); Sloughter et al. (2011), ensemble copula coupling (ECC; Schefzik 2011) and the postprocessing method proposed by Pinson (2011), all of which are directed at the bivariate postprocessing of ensemble forecasts of wind vectors. The Appendix describes our verification methods.

\section{Ensemble model output statistics for wind vectors}

A wind vector is determined by wind speed and wind direction, or by its zonal (west-east) and meridional (north-south) components, which we denote by $u$ and $v$, respectively. We now develop an ensemble model output statistics (EMOS) method for wind vectors, where the postprocessed probabilistic forecast takes the form of a bivariate probability density function. The method is tailored to ensembles with relatively few members, such as the eight-member University of Washington Mesoscale Ensemble (UWME; Eckel and Mass 2005), and we illustrate it using forecast and observation data from this ensemble.

\subsection{Bivariate normal distribution}

Our EMOS postprocessed forecast takes the form of an elliptically symmetric, bivariate normal probability density function for the wind vector $(u, v)$, with the parameters of this distribution being specified in terms of the ensemble forecast. The analytic form of the bivariate normal probability density function is

$$
\begin{aligned}
& f(u, v)=\frac{1}{2 \pi \sigma_{u} \sigma_{v} \sqrt{1-\rho_{u v}^{2}}} \\
& \times \exp \left(-\frac{1}{2\left(1-\rho_{u v}^{2}\right)}\left(\frac{\left(u-\mu_{u}\right)^{2}}{\sigma_{u}^{2}}-2 \rho_{u v} \frac{\left(u-\mu_{u}\right)\left(v-\mu_{v}\right)}{\sigma_{u} \sigma_{v}}+\frac{\left(v-\mu_{v}\right)^{2}}{\sigma_{v}^{2}}\right)\right) .
\end{aligned}
$$

The task now is to specify the five parameters in (1), namely the mean values, $\mu_{u}$ and $\mu_{v}$, of the wind vector components $u$ and $v$, the corresponding marginal variances, $\sigma_{u}^{2}$ and $\sigma_{v}^{2}$, respectively, and the correlation coefficient, $\rho_{u v}$, between the wind components, in their dependence on the ensemble forecast.

Bivariate normal density forecasts for wind vectors have also been proposed by Gneiting et al. (2008), though in very crude form. The ingenious method of Pinson (2011) estimates a dilation and translation of an ensemble forecast of wind vectors based on bivariate normal densities, and our approach is very similar in its treatment of the mean and variance parameters. However, major differences between the approach of Pinson (2011) and our method lie in the form of the postprocessed forecast, which is a probability density function in our case, rather than a dilated and translated ensemble, and in the explicit modeling of the correlation coefficient $\rho_{u v}$ in our method. For a more detailed comparison and recommendations for a judicious choice of the most appropriate method, given any particular ensemble and task at hand, we refer to Section 4. 
In the description that follows, we consider a general ensemble with $m$ members, and denote the individual wind vector forecasts by $\left(u_{1}, v_{1}\right), \ldots,\left(u_{m}, v_{m}\right)$, respectively.

\subsection{Means}

In our standard implementation of the bivariate EMOS technique, the means $\mu_{u}$ and $\mu_{v}$ are biascorrected versions of the respective ensemble means, in that

$$
\mu_{u}=a_{u}+b_{u} \bar{u} \quad \text { and } \quad \mu_{v}=a_{v}+b_{v} \bar{v},
$$

where $\bar{u}=\frac{1}{m} \sum_{i=1}^{m} u_{i}$ and $\bar{v}=\frac{1}{m} \sum_{i=1}^{m} v_{i}$. The bias correction parameters $a_{u}, b_{u}, a_{v}$ and $b_{v}$ are estimated from training data, in ways described below. In a slightly more ambitious implementation, the mean components $\mu_{u}$ and $\mu_{v}$ are affine functions of the individual ensemble member forecasts, namely

$$
\mu_{u}=a_{u}+b_{u, 1} u_{1}+\cdots+b_{u, m} u_{m} \quad \text { and } \quad \mu_{v}=a_{v}+b_{v, 1} v_{1}+\cdots+b_{v, m} v_{m}
$$

where the regression parameters $a_{u}, b_{u, 1}, \ldots, b_{u, m}, a_{v}$ and $b_{v, 1}, \ldots, b_{v, m}$ are estimated from training data. This general version applies to ensembles with non-exchangeable members only and reduces to the standard version when $b_{u, 1}=\cdots=b_{u, m}=\frac{1}{m}$ and $b_{v, 1}=\cdots=b_{v, m}=\frac{1}{m}$. In our experiences with the UWME, which has non-exchangeable members, the general version gave only very slightly improved predictive performance, and so we report results for the standard implementation (2) only.

\subsection{Variances}

We specify the marginal variances $\sigma_{u}^{2}$ and $\sigma_{v}^{2}$ of the bivariate normal density forecast (1) as affine functions of the respective ensemble variances, in that

$$
\sigma_{u}^{2}=c_{u}+d_{u} s_{u}^{2} \quad \text { and } \quad \sigma_{v}^{2}=c_{v}+d_{v} s_{v}^{2}
$$

where $s_{u}^{2}=\frac{1}{m} \sum_{i=1}^{m}\left(u_{i}-\bar{u}\right)^{2}$ and $s_{v}^{2}=\frac{1}{m} \sum_{i=1}^{m}\left(v_{i}-\bar{v}\right)^{2}$. The dispersion correction parameters $c_{u}$, $d_{u}, c_{v}$ and $d_{v}$ are estimated from training data, as described below. To guarantee the nonnegativity of the variances, we constrain the parameters to be nonnegative, using the technique described by Thorarinsdottir and Gneiting (2010).

\subsection{Correlation coefficient}

The key characteristic and major innovation in our work is the explicit modeling of the correlation coefficient $\rho_{u v}$ of the postprocessed bivariate normal density forecast (1).

To motivate our specification of $\rho_{u v}$, we consider ensemble forecast and observation data from the UWME in calendar year 2007, comprising a total of 23,250 forecasts cases at 79 meteorological stations in the Pacific Northwest. Figure 2 distinguishes nine sectors for $(u, v)$ wind vector forecasts and observations. Figure 3 shows wind vector observations in 2007, conditionally on the ensemble mean wind vector falling into any given sector. The conditional distributions are partly elliptically contoured, particularly in the first sector, and partly skewed, with an orientation that depends strongly on the sector, and they reflect the discretized nature of wind observations, as discussed in more detail in Section 3 . 


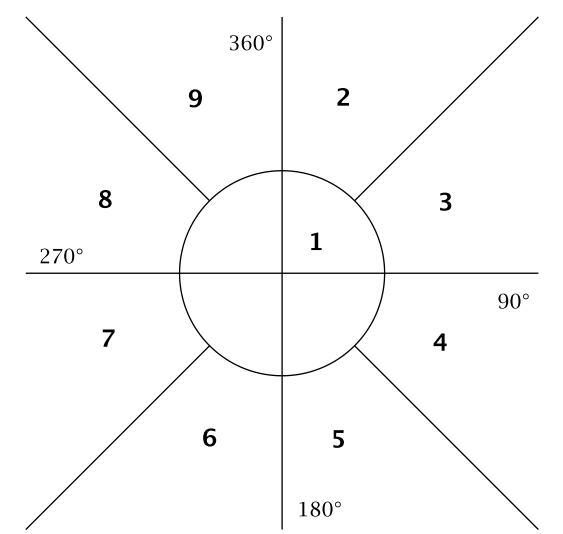

Figure 2: Sectors for wind vector forecasts and observations in the $(u, v)$ plane. Sector 1 is the circular region that is centered at the origin and corresponds to wind speeds less than or equal to two meters per second. Sectors 2-9 are assigned clockwise, with Sectors 2-3 corresponding to a south-westerly, Sectors 4-5 a north-westerly, Sectors 6-7 a north-easterly, and Sectors 8-9 a south-easterly wind.

The left-hand panel in Figure 4 plots the correlation coefficient between the wind components in the scatter plots in Figure 3 as a function of the wind directions that correspond to the centers of sectors 2-9. The panel demonstrates that the ensemble mean wind direction ought to have a profound influence on the correlation coefficient $\rho_{u v}$ in the postprocessed EMOS density forecast (1). Thus, we model the correlation coefficient $\rho_{u v}$ as a trigonometric function of the ensemble mean wind direction, $\theta$, measured in degrees, in that

$$
\rho_{u v}=r \cos \left(\frac{2 \pi}{360}(k \theta+\varphi)\right)+s
$$

where the parameters $r, s, k$ and $\varphi$ are estimated from training data, in ways described below. The coefficients $r$ and $s$ concern the overall magnitude of the correlation coefficient and need to satisfy $|r|+|s| \leq 1$. The parameter $k$ corresponds to the number of periods of the trigonometric function, which we constrain to be either $k=1,2$ or 3 , and the direction $\varphi$ encodes phase information.

We apply the correlation model (4) to forecasts with ensemble mean vectors in all nine wind sectors, including the first sector. Alternatively, if the ensemble mean wind vector falls into the central first sector, one can take $\rho_{u v}$ to be equal to the empirical correlation coefficient in the corresponding scatterplot. In our experiences with the UWME, the two approaches resulted in nearly identical predictive performance.

\subsection{Estimation}

Our EMOS postprocessed density forecast for a wind vector takes the form of the bivariate normal probability density (1), where the relationships of $\mu_{u}, \mu_{v}, \sigma_{u}^{2}, \sigma_{v}^{2}$ and $\rho_{u v}$ to the raw ensemble forecast are specified in equations (2), (3) and (4). It remains to estimate the parameters that govern these equations, and we address this task in three phases.

Before describing the phases of our estimation scheme, it is worth noting that we follow Thorarinsdottir and Gneiting (2010) and consider two distinct variants, which we call the Regional EMOS and the Local EMOS method, respectively. In the Regional EMOS method, only one set of 

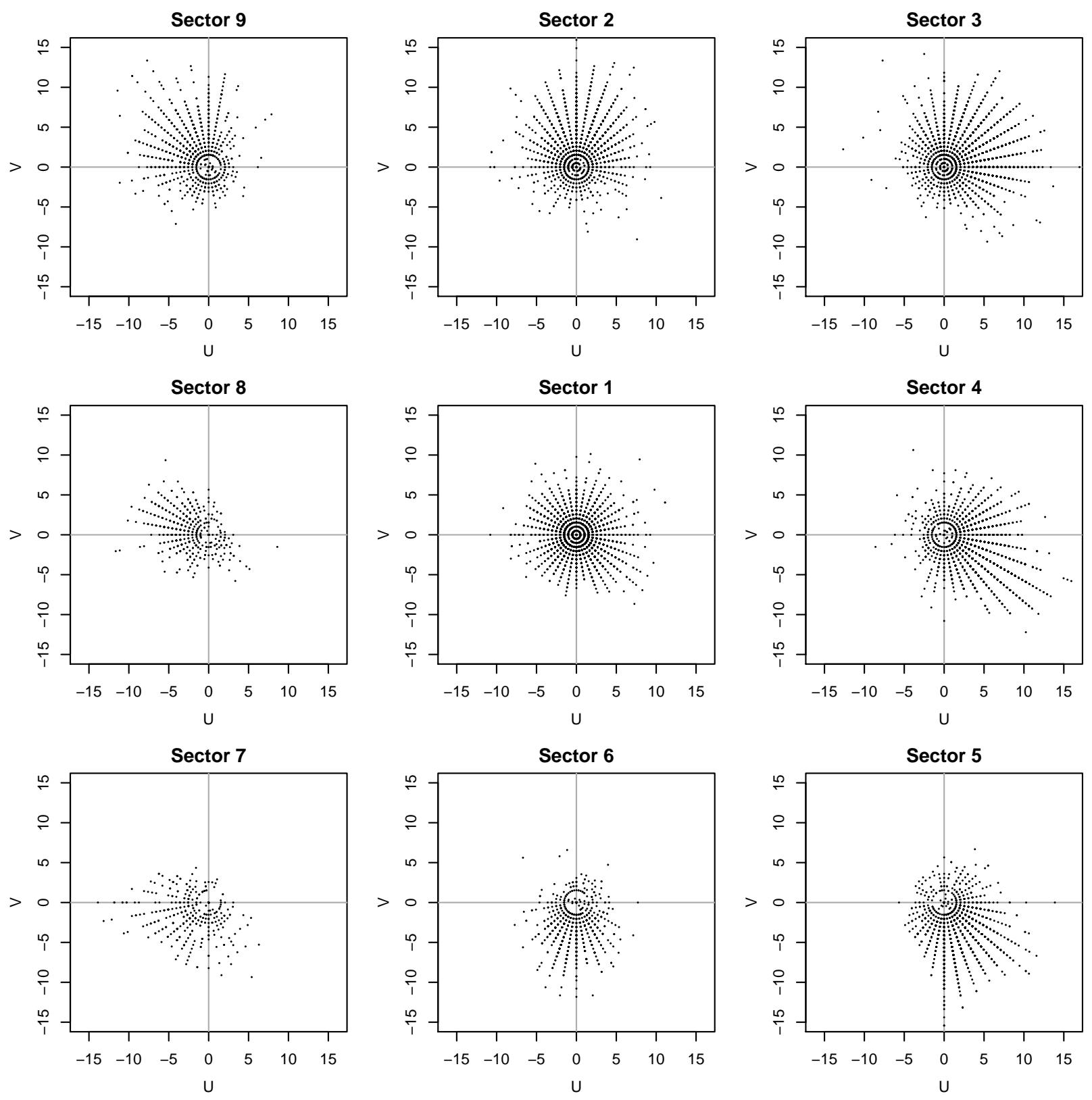

Figure 3: Wind vector observations over the Pacific Northwest in 2007, conditional on the ensemble mean forecast falling into one of the sectors defined in Figure 2. The unit for the wind components is meters per second. 

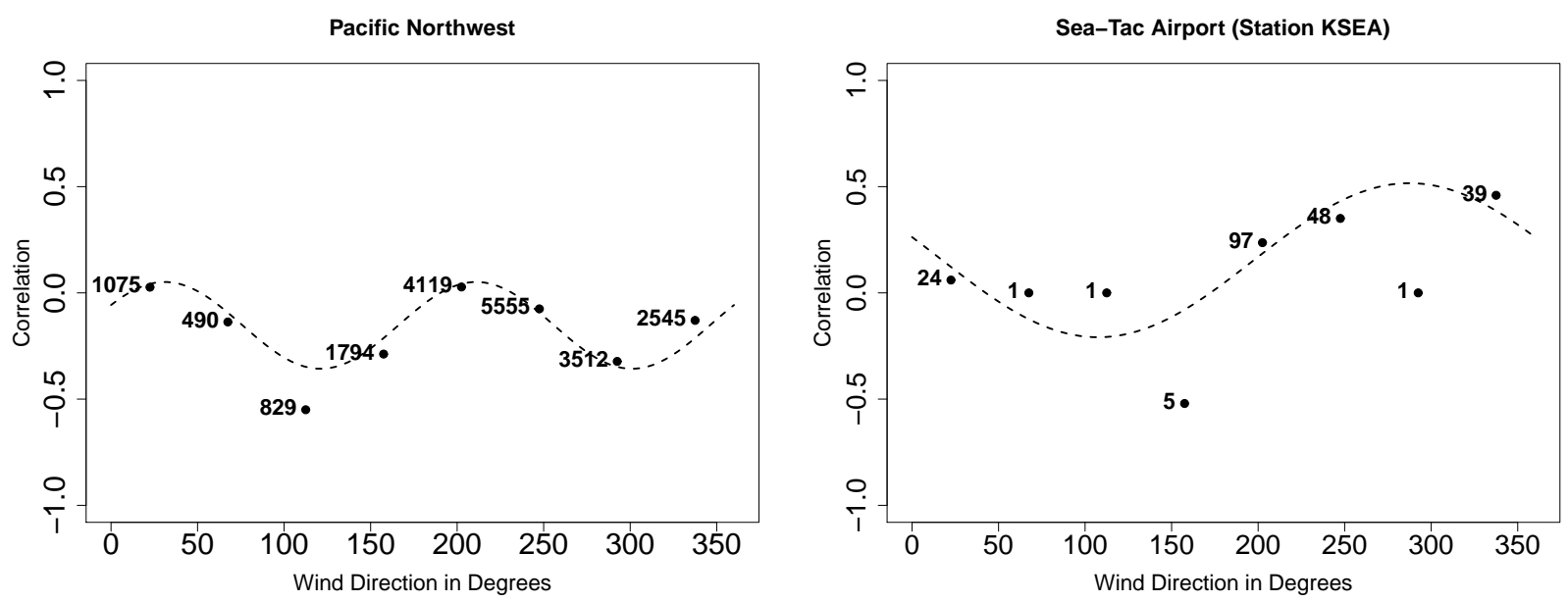

Figure 4: Left: Correlation coefficient between the wind components in the scatter plots in Figure 3 as a function of the wind direction that corresponds to the center of the sector. The correlation coefficients and observation counts correspond to sectors $2-9$, respectively. The dashed curve shows the correlation model (4) as fitted by the weighted least squares method. Right: Same as left, but considering data from Sea-Tac Airport only.

parameters is estimated and used to produce forecasts over the entire ensemble domain, such as the Pacific Northwest for the UWME. Training sets thus comprise data from all stations. In contrast, the Local EMOS method uses training data from the station at hand only, and thus obtains a distinct set of parameters for each station.

We now turn to the first phase of our estimation scheme, in which we fit the correlation model (4). We do this offline, once and for all, based on historic, out-of-sample forecast and observation data. Specifically, for the UWME, we use data from calendar year 2007 to form the conditional scatterplots in Figure 3 and compute and plot the corresponding empirical correlation coefficients, as illustrated in Figure 4. We then decide about a suitable value for the number of cycles, $k$, and fit the remaining parameters, $r, s$ and $\varphi$, of the correlation model (4) by a weighted non-linear least squares technique, using the R function NLS with the weights being proportional to the number of observations in the sectors ( $\mathrm{R}$ Development Core Team, 2011). The use of a weighted least squares technique is critical, particularly for the Local EMOS technique, as local wind patterns may result in very few observations being available in any given sector. This first phase of the estimation is done once and for all, using historic data from 2007, and the fitted correlation model is applied throughout calendar year 2008, which we took as our test period.

The left-hand panel in Figure 4 shows the fitted correlation model for the Regional EMOS method, where we chose $k=2$ and obtained weighted least squares estimates of $r=0.20$, $s=-0.15$ and $\varphi=-61.9$ degrees, respectively. As noted, the Regional EMOS method uses these parameters throughout the UWME domain and throughout the test period in calendar year 2008. The right-hand panel shows the Local EMOS correlation model at Seattle-Tacoma (Sea-Tac) Airport, where we took $k=1$ and obtained weighted least squares estimates of $r=0.24, s=0.07$ and $\varphi=70.5$ degrees, respectively. The fitted Local EMOS correlation models at the remaining stations in the UWME domain are provided and illustrated in the appendix of Schuhen (2011).

In contrast to the first phase of our estimation scheme, the second and third stages proceed on- 
line, that is, they use rolling training periods consisting of data from the recent past. The Regional EMOS method uses all available data from the Pacific Northwest from the last $n$ days prior to the forecast being made. Missing data are simply omitted from the training set. For the Local EMOS method, the training period comprises data from the station at hand from the $n$ most recent days where forecasts and observations were available. In either case, we talk of a sliding $n$-day training period.

In the second phase of our estimation scheme, the parameters $a_{u}, b_{u}, a_{v}$ and $b_{v}$ in the specification (2) of the mean vector are estimated from the training data by standard linear least squares regression. In the third phase, the parameters $c_{u}, d_{u}, c_{v}$ and $d_{v}$ in the specification (3) of the marginal variances are estimated on the same rolling training period by the maximum likelihood technique, with all other parameters being held fixed. In other words, we maximize the logarithm of the likelihood function, namely

$$
l\left(c_{u}, d_{u}, c_{v}, d_{v}\right)=\sum_{(x, t)} \log f^{(x, t)}\left(c_{u}, d_{u}, c_{v}, d_{v}\right),
$$

as a function of the parameters $c_{u}, d_{u}, c_{v}$ and $d_{v}$ in the variance model (3), which are all constrained to be nonnegative. The sum in the log likelihood function extends over all locations $x$ and times $t$ for which there are data in the training set. Any single term of the form $f^{(x, t)}\left(c_{u}, d_{u}, c_{v}, d_{v}\right)$ refers to the bivariate normal density (1) evaluated at the verifying values $u=u^{(x, t)}$ and $v=v^{(x, t)}$, with $\mu_{u}$, $\mu_{v}$ and $\rho_{u v}$ set at the numerical values implied by the mean model (2) and the correlation model (4), based on the parameter estimates from the first two phases of our estimation scheme, and putting $\sigma_{u}^{2}=c_{u}+d_{u} s_{u}^{2 \mid(x, t)}$ and $\sigma_{v}^{2}=c_{v}+d_{v} s_{v}^{2 \mid(x, t)}$, where $s_{u}^{2 \mid(x, t)}$ and $s_{v}^{2 \mid(x, t)}$ denote the ensemble variances at location $x$ and valid time $t$, respectively. The optimization is performed numerically with the OPTIM function in R, using the Broyden-Fletcher-Goldfarb-Shanno algorithm with initial values provided by the previous day's estimates.

The interpretation of the right-hand side of (5) as a log likelihood function is valid only if the forecast errors are independent between times and locations. While this is usually not the case, an alternative interpretation as a mean logarithmic score permits us to view the estimates as optimum score estimates, which are tailored to the estimation of forecasting models (Gneiting et al., 2005).

\subsection{Example}

Figure 5]and Table 1 illustrate the postprocessed Local and Regional EMOS density forecasts of the surface wind vector at Sea-Tac Airport, valid October 20, 2008 at 00 UTC, at a prediction horizon of 48 hours. Thus, the forecast concerns the same valid time and the same prediction horizon as the Local EMOS forecasts illustrated in Figure 1, where the station at Sea-Tac Airport is located in the south-east Puget Sound area. The postprocessed density forecasts correct for the biases and underdispersion in the raw UWME. The Local EMOS forecast retains the positive correlation structure in the raw ensemble and is sharper than the Regional EMOS forecast.

\section{Case study: Forecasting surface wind vectors over the Pacific Northwest}

We now consider the out-of-sample predictive performance of our two-dimensional EMOS technique in a case study for wind vector forecasts over the North American Pacific Northwest in 2008, 

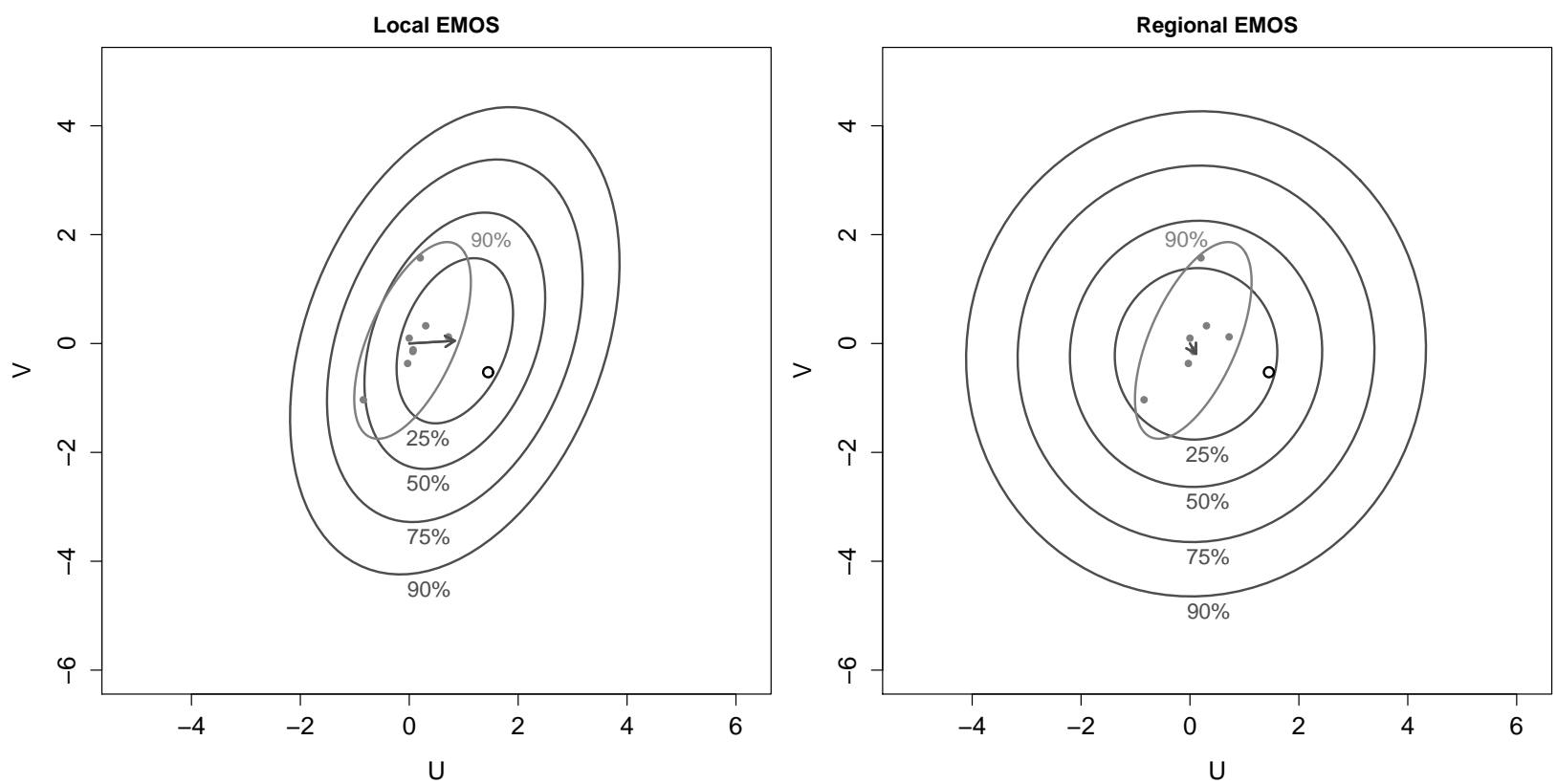

Figure 5: Contour plot of the postprocessed Local EMOS (left) and Regional EMOS (right) density forecasts, along with the raw ensemble forecast, of the surface wind vector at Sea-Tac Airport, valid October 20, 2008 at 00 UTC, at a prediction horizon of 48 hours. The eight members of the University of Washington Mesoscale Ensemble (UWME; Eckel and Mass 2005) are shown as gray dots, along with the 90\% prediction ellipse, which is based on a bivariate Gaussian fit to the ensemble values. The $25 \%, 50 \%, 75 \%$ and $90 \%$ prediction ellipses and the mean vector for the postprocessed EMOS density forecast are shown in dark gray. The verifying wind vector is represented by the small black circle at $(u, v)=(1.45,-0.53)$. The units are in meters per second.

Table 1: Predictive means, variances and correlation for the postprocessed Local and Regional EMOS density forecasts in Figure 5 of the surface wind vector at Sea-Tac Airport, valid October 20, 2008 at 00 UTC, at a prediction horizon of 48 hours. The parameters refer to the general bivariate normal probability density (1), with the wind components represented in meters per second.

\begin{tabular}{lcc}
\hline Method & Local EMOS & Regional EMOS \\
\hline$\mu_{u}$ & 0.84 & 0.11 \\
$\mu_{v}$ & 0.05 & -0.19 \\
$\sigma_{u}^{2}$ & 1.99 & 3.87 \\
$\sigma_{v}^{2}$ & 4.00 & 4.31 \\
$\rho_{u v}$ & 0.33 & -0.02 \\
\hline
\end{tabular}


based on the University of Washington Mesoscale Ensemble (UWME; Eckel and Mass 2005). We compare to the raw ensemble forecast and various reference techniques, such as ensemble copula coupling, and assess the performance of the bivariate EMOS technique when forecasts of wind speed are desired only.

\subsection{University of Washington Mesoscale Ensemble}

In our case study, the test set consists of forecasts of surface (10 meter) wind vectors based on the University of Washington Mesoscale Ensemble (UWME; Eckel and Mass 2005) with valid date in calendar year 2008, at a prediction horizon of 48 hours. The UWME is an eight-member multianalysis ensemble then based on the Fifth-Generation Penn State/NCAR Mesoscale Model (MM5) with initial and lateral boundary conditions obtained from operational centers around the world. The forecasts are made on a $12 \mathrm{~km}$ grid and the region covered is the Pacific Northwest region of Western North America, including the US states of Washington, Oregon and Idaho, as well as the southern part of the Canadian province of British Columbia. The forecasts were bilinearly interpolated from the four surrounding grid points to the observation locations and rotated to match the true direction at each station.

Surface wind vector observations were provided by the weather observation stations in the Automated Surface Observing System network (National Weather Service, 1998). The vector wind quantity studied here is horizontal instantaneous surface wind, where 'instantaneous' means that the wind was measured and averaged over the last two minutes before the valid time at 00 UTC. The wind vector observations were recorded as wind speed and wind direction, where wind speed was rounded to the nearest whole knot, where a knot equals 0.5144 meters per second, while values below two knots were recorded as zero. The observations are thus discretized, as is easily recognizable in Figure 3, Quality control procedures as described in Baars (2005) were applied to the entire data set, removing dates and locations with any missing forecasts or observations.

For calendar year 2008, 19,282 pairs of ensemble forecasts and observations were available on 291 distinct days and at 79 distinct observation locations. Additional data from the years 2006 and 2007 were used to provide an appropriate rolling training period for all days in 2008, for the first phase estimation of the correlation model (4) and to establish the optimal length of the rolling training period. Further information about the UWME, now using the WRF mesoscale model, as well as real time forecasts and observations, can be found online at http://www . atmos . washington . edu/ ens/uwme.cgi.

\subsection{Training periods for Regional and Local EMOS}

As noted, we distinguish Regional and Local EMOS forecasts. The Regional EMOS method uses all available training data to estimate a single set of parameters that is used throughout the Pacific Northwest domain, and can be used directly on the model grid as well. The Local EMOS technique uses training data from the station at hand only to obtain a distinct set of parameters at each station. Thus, the method applies at observation stations only, and can not be used directly on the model grid.

To determine a suitable value of the length $n$ of the rolling training period for phases two and three of our estimation scheme, as described in Section 2, we considered experiments with wind vector forecasts based on the UWME in calendar year 2007. In these experiments, phase one of 

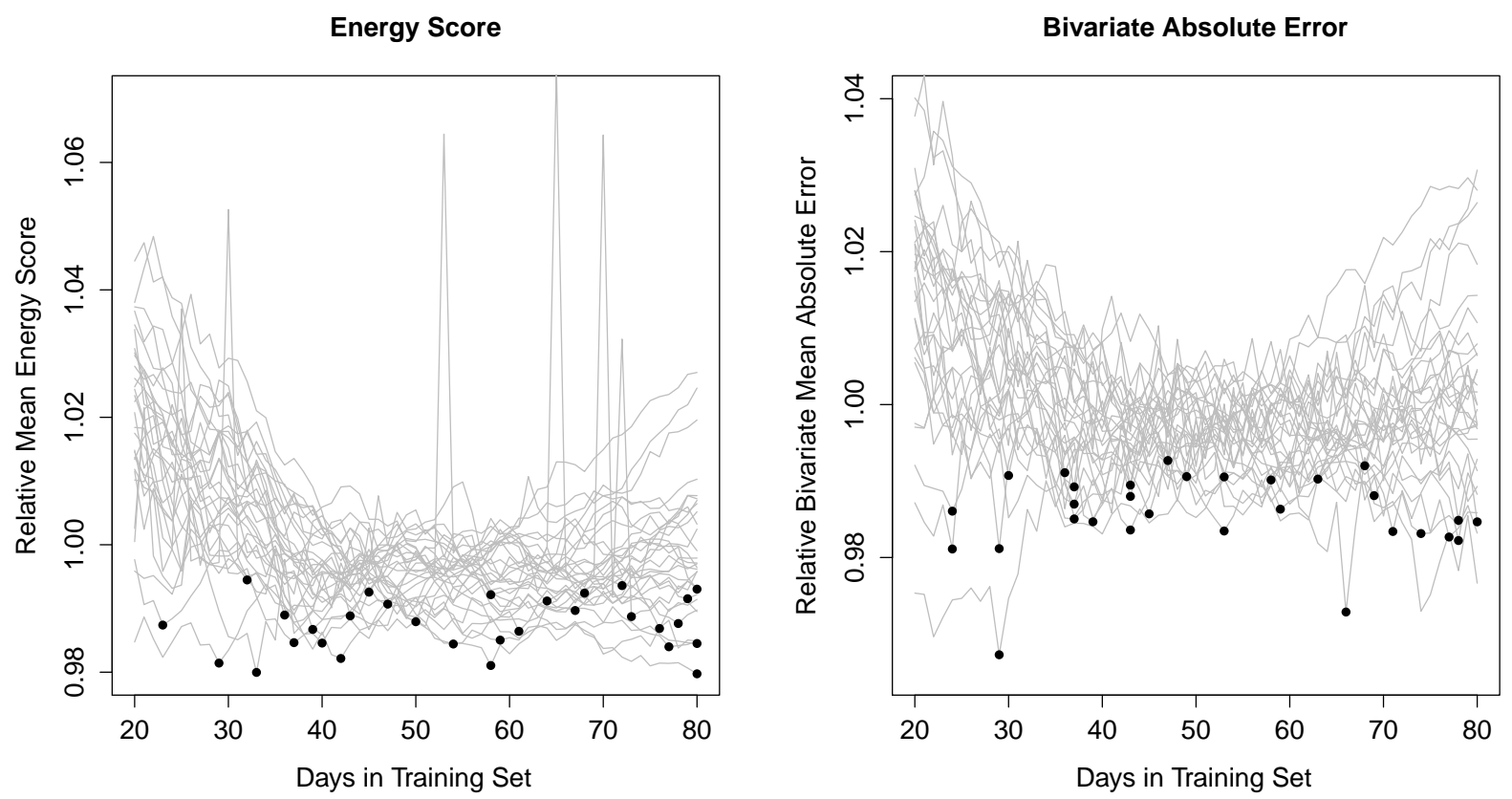

Figure 6: Predictive performance of the Local EMOS method in calendar year 2007 as a function of the length of the rolling training period in terms of the relative mean energy score (left) and the relative bivariate mean absolute error (right). Each curve corresponds to an observation station in Washington state, with the dot indicating the lowest value of the performance measure, relative to the average value among the lengths considered.

the estimation scheme used (here, in-sample) data from 2007, while phases two and three were done on a rolling training period of length $n$ days. The predictive performance was evaluated using the mean energy score and the bivariate mean absolute error, as described in the Appendix. For the Regional EMOS method, these metrics differed by less than a half percent as the length $n$ of the rolling training period varied between 25 and 60 . Figure 6 summarizes the results for the Local EMOS method, where we follow Thorarinsdottir and Gneiting (2010) and consider the observational locations in Washington state. Based on these results, our case study in calendar 2008 uses a rolling training period of length $n=30$ days for the Regional EMOS method, and of length $n=40$ days for the Local EMOS technique. However, training periods of any length between $n=20$ and $n=80$ days work well, and the predictive performance of the EMOS methods is insensitive to this choice.

\subsection{Reference forecasts}

We compare the postprocessed Regional and Local EMOS forecasts to the raw UWME forecast, as well as to postprocessed reference forecasts, as described now. For all reference forecasts, we distinguish Regional and Local methods, using rolling training periods of the most recent available $n=30$ and $n=40$ days, respectively.

A natural reference standard is the Independent EMOS technique, which applies the standard EMOS or heterogeneous Gaussian regression technique of Gneiting et al. (2005) to each of the 
vector wind components $u$ and $v$ individually, and then combines them under the assumption of independence. This results in a postprocessed bivariate normal density forecast of the form (1) with the critical correlation parameter $\rho_{u v}$ constrained to be zero, but with the means and variances for $u$ and $v$ being essentially identical to those in the bivariate EMOS method, except for minor differences due to the slightly differing estimation schemes. In particular, the bivariate EMOS method and the Independent EMOS technique yield essentially identical deterministic, bivariate mean and median wind vector forecasts, even though the respective bivariate predictive densities may differ substantially.

The Ensemble Copula Coupling (ECC) method, originally hinted at by Bremnes (2007) and Krzysztofowicz and Toth (2008) and recently investigated and developed by Schefzik (2011), is a tool for restoring a raw ensemble's flow-dependent rank dependence structure in individually postprocessed predictive distributions. Here, we describe and apply the method in the context of the Independent EMOS technique and a raw ensemble with $m$ members. At any given location and prediction horizon, let $u_{1}^{*}, \ldots, u_{m}^{*}$ and $v_{1}^{*}, \ldots, v_{m}^{*}$ denote samples from the individually postprocessed univariate EMOS distributions. As the postprocessed distributions for the wind vector components are univariate Gaussian, these are simply random numbers drawn from a univariate normal density. Let $u_{1}, \ldots, u_{m}$ and $v_{1}, \ldots, v_{m}$ denote the raw ensemble values for the respective wind components, and let $\tau_{u}$ and $\tau_{v}$ be permutations of the numbers $1, \ldots, m$ such that

$$
u_{\tau_{u}(1)} \leq u_{\tau_{u}(2)} \leq \cdots \leq u_{\tau_{u}(m)} \quad \text { and } \quad v_{\tau_{v}(1)} \leq v_{\tau_{v}(2)} \leq \cdots \leq v_{\tau_{v}(m)}
$$

with any ties resolved at random. The ECC ensemble then consists of the $m$ wind vectors

$$
\left(u_{\tau_{u}(1)}^{*}, v_{\tau_{v}(1)}^{*}\right), \ldots,\left(u_{\tau_{u}(m)}^{*}, v_{\tau_{v}(m)}^{*}\right) .
$$

Thus, the ECC ensemble inherits and honors the raw ensemble's rank dependence structure. For example, if the first raw ensemble member shows the second lowest $u$ component and the third highest $v$ component among the raw ensemble members, then the same property holds true for the ECC ensemble.

Schefzik (2011) provides a detailed discussion of the ECC technique and exposes its ties to copulas (Schölzel and Friederichs, 2008). The wind vector postprocessing technique recently proposed by Pinson (2011) can be interpreted as a particularly attractive variant, where the values

$u_{1}^{*}, \ldots, u_{m}^{*}$ are constructed as a translation and dilation of the raw ensemble values $u_{1}, \ldots, u_{m}$, and $v_{1}^{*}, \ldots, v_{m}^{*}$ as a translation and dilation of $v_{1}, \ldots, v_{m}$, respectively, rather than being drawn at random. Consequently, the postprocessed ensemble forecast retains both the raw ensemble's bivariate Spearman rank correlation and its (standard) Pearson product moment correlation structure.

Finally, we consider an Error Dressing ensemble as proposed by Gneiting et al. (2008) in the spirit of the work of Roulston and Smith (2003), where we dress the UWME mean forecast with 35 error vectors from the corresponding training set.

\subsection{Results over the Pacific Northwest}

We now give verification results aggregated over our test period, the calendar year 2008, and the Pacific Northwest domain of the UWME. For details about the verification techniques method, which include the multivariate rank histogram, as proposed by Gneiting et al. (2008) for calibration 

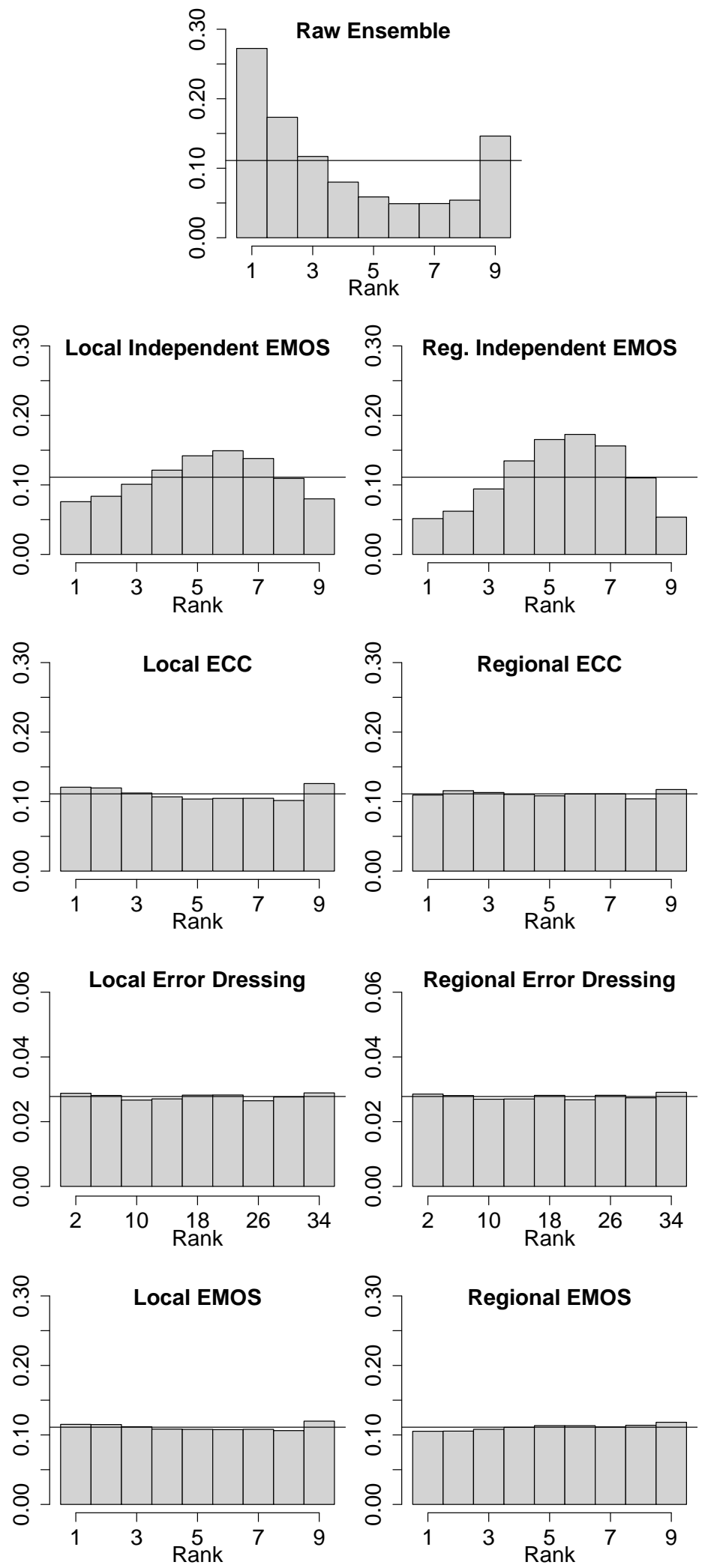

Figure 7: Multivariate rank histograms for raw and postprocessed ensemble forecasts of surface wind vectors, aggregated over calendar year 2008 and the Pacific Northwest. 
Table 2: Predictive performance of forecasts of surface wind vectors in terms of the mean energy score (ES) and the bivariate mean absolute error (bMAE), both in meters per second, and the reliability index $\Delta$ for the multivariate rank histogram, aggregated over calendar year 2008 and the Pacific Northwest.

\begin{tabular}{lccc}
\hline Method & ES & bMAE & $\Delta$ \\
\hline Raw Ensemble & 2.47 & 3.01 & 0.53 \\
\hline Regional Independent EMOS & 2.43 & 2.79 & 0.37 \\
Regional ECC & 2.33 & 3.00 & 0.02 \\
Regional Error Dressing & 2.19 & 3.01 & 0.01 \\
Regional EMOS & 2.01 & 2.80 & 0.03 \\
\hline Local Independent EMOS & 2.28 & 2.60 & 0.21 \\
Local ECC & 2.16 & 2.78 & 0.07 \\
Local Error Dressing & 2.07 & 2.87 & 0.01 \\
Local EMOS & 1.87 & 2.61 & 0.03 \\
\hline
\end{tabular}

checks, and proper scoring rules (Gneiting and Raftery, 2007), are given in the Appendix. All scores used are negatively oriented, that is, the smaller the better.

Figure 7 shows multivariate rank histograms for the raw ensemble forecast and Regional and Local versions of the Independent EMOS, ECC, Error Dressing and (bivariate) EMOS techniques. The raw ensemble forecast shows a U-shaped rank histogram, thus indicating underdispersion. In contrast, the Independent EMOS forecasts are overdispersed, as they neglect to take dependencies between the wind vector components into account, which can be corrected for with the ECC technique. In addition to the ECC method, the Error Dressing and (bivariate) EMOS techniques show uniform rank histograms, as expected from a calibrated forecast.

Table 2 provides numerical summary measures of the predictive performance, with the reliability index $\Delta$ for the multivariate rank histogram confirming the visual impression in Figure 7 . The energy score is a direct analogue of the continuous ranked probability score for univariate quantities that provides an overall assessment of the quality of a probabilistic forecast, addressing both calibration and sharpness. The bivariate absolute error generalizes the absolute error and assesses deterministic forecast skill.

Not surprisingly, the Local approaches outperform the Regional approaches, both in terms of the mean energy score and the bivariate mean absolute error. Also, the postprocessed Independent EMOS forecasts show lower scores than the raw forecast. The Independent EMOS and the (bivariate) EMOS forecasts have nearly identical bivariate mean absolute error, at a substantially lower value than for the other types of forecasts, including the ECC approach. This effect can be attributed to a discretization effect, in that the ECC technique turns the Independent EMOS density forecast into a discrete ensemble forecast. However, the ECC approach improves on the Independent EMOS forecast in terms of the energy score, as it honors the raw ensemble's flow-dependent bivariate dependence structure. The Error Dressing technique also shows good probabilistic forecast skill, as evidenced by a low energy score, even though it is unable to match the scores of the (bivariate) EMOS method, which performs the best. When compared to the raw ensemble, the postprocessed Local EMOS forecast reduces the mean energy score from 2.47 to 1.87 meters per second. 


\subsection{Results at Sea-Tac Airport}

Next we consider forecasts at the observation station at Sea-Tac Airport, with summary measures of the predictive performance in calendar year 2008 being provided in Table 3 . The results mimic those for the Pacific Northwest. The Local EMOS forecast shows the highest probabilistic forecast skill, as quantified by the energy score, which decreases to 1.94 meters per second, as compared to 2.25 meters per second for the raw ensemble.

For an illustration and explanation of how and to what extent the Local EMOS technique succeeds in improving the raw ensemble forecast at Sea-Tac Airport, consider the display in Figure 8 , which is a bivariate variant of the marginal calibration diagram proposed by Gneiting et al. (2007). Essentially, a marginal calibration diagram compares the observed climatology to the climatology incurred by the forecasts. In our display we plot, for each day for which data are available, the observed wind vector, perturbed very slightly in order to honor the undiscretized distribution and improve readability, a randomly chosen member of the UWME raw ensemble, and a wind vector sampled from the postprocessed, bivariate normal Local EMOS density forecast. Again we observe that the raw ensemble is underdispersive and fails to predict any extreme wind vectors. The postprocessed Local EMOS forecast corrects for the underdispersion, thus leading to substantially improved probabilistic forecast skill, as evidenced by the energy score. However, the raw ensemble does not show any recognizable biases, and so the improvement in deterministic forecast skill is minor.

\subsection{Results for wind speed}

In addition to producing calibrated and sharp forecasts of wind vectors, the bivariate EMOS method can be used to predict wind speeds, and so can be compared to the technique of Thorarinsdottir and Gneiting (2010), which is custom tailored to this task. As wind speed is a nonnegative quantity, we employ truncated normal predictive distributions, using an estimation scheme that is based on optimum score estimation, both in Regional and Local versions, where we use rolling training periods comprising the most recent $n=30$ and $n=40$ days available, respectively. In what follows, we refer to the method of Thorarinsdottir and Gneiting (2010) as wind speed EMOS.

To generate probabilistic forecasts of wind speed from the bivariate EMOS forecast, we sample one hundred wind vectors from the bivariate predictive distribution, and compute the Euclidean norm of each vector, thereby obtaining a discrete forecast ensemble of size $m=100$ for wind speed. Table 4 compares the predictive performance of this approach to that of the wind speed EMOS technique. As noted, the wind speed observations are strongly discrete, with wind speeds below two knots recorded as zero, which applies to about $14 \%$ of the observations in the test period. From the perspective of wind vectors, these observations tend to fall into the center of the respective predictive distribution, and so the effect of the discretization is weak. From the perspective of wind speeds, an observed value of zero is right at the boundary of the climatological range, and so the effect is nonnegligible. To account for it, we replace every observation of zero by a number drawn uniformly and at random between zero and two knots, or between zero and 1.03 meters per seconds, when computing the corresponding continuous ranked probability score or absolute error. The bivariate EMOS technique, particularly in its Local version, nearly matches the predictive performance of the specialized wind speed EMOS technique of Thorarinsdottir and Gneiting (2010). 
Table 3: Predictive performance of forecasts of surface wind vectors at Sea-Tac Airport in calendar year 2008 in terms of the mean energy score (ES) and the bivariate mean absolute error (bMAE), both in meters per second, and the reliability index $\Delta$ for the multivariate rank histogram.

\begin{tabular}{lccc}
\hline Method & ES & bMAE & $\Delta$ \\
\hline Raw Ensemble & 2.25 & 2.77 & 0.54 \\
\hline Regional Independent EMOS & 2.41 & 2.89 & 0.29 \\
Regional ECC & 2.37 & 3.06 & 0.23 \\
Regional Error Dressing & 2.12 & 2.92 & 0.07 \\
Regional EMOS & 2.06 & 2.90 & 0.24 \\
\hline Local Independent EMOS & 2.40 & 2.73 & 0.22 \\
Local ECC & 2.21 & 2.87 & 0.22 \\
Local Error Dressing & 1.98 & 2.75 & 0.02 \\
Local EMOS & 1.94 & 2.74 & 0.09 \\
\hline
\end{tabular}

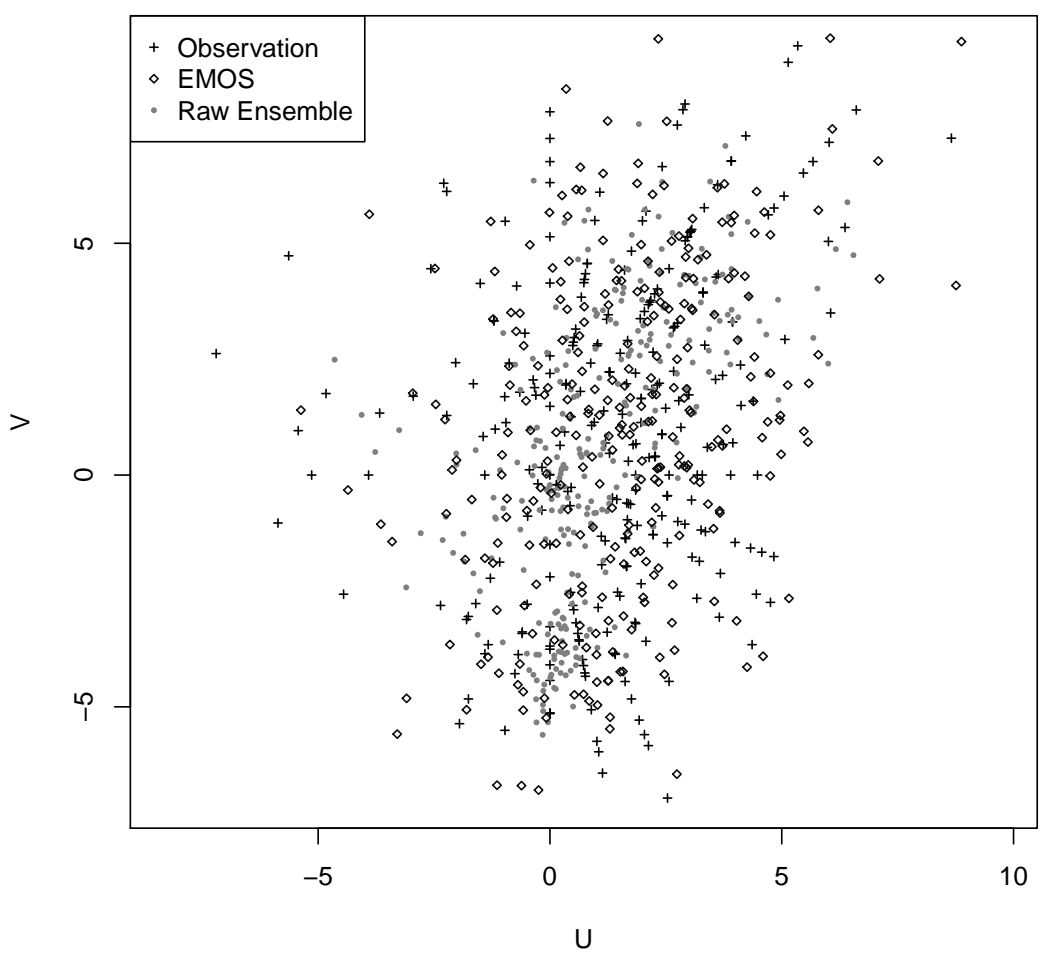

Figure 8: Marginal calibration diagram for forecasts of surface wind vectors at Sea-Tac Airport in calendar year 2008. For each day available, the plot shows the observed wind vector, a randomly chosen member of the raw ensemble, and a wind vector sampled from the postprocessed Local EMOS density forecast. 
Table 4: Predictive performance of forecasts of wind speed in terms of the mean continuous ranked probability score (CRPS) and the mean absolute error (MAE), in meters per second, averaged over calendar year 2008 and the Pacific Northwest.

\begin{tabular}{lcc}
\hline Method & CRPS & MAE \\
\hline Raw Ensemble & 1.34 & 1.68 \\
\hline Regional Wind Speed EMOS & 1.11 & 1.57 \\
Regional EMOS & 1.15 & 1.63 \\
\hline Local Wind Speed EMOS & 1.08 & 1.50 \\
Local EMOS & 1.07 & 1.51 \\
\hline
\end{tabular}

\section{Discussion}

In this paper, we have proposed a bivariate EMOS approach to the statistical postprocessing of ensemble forecasts of wind vectors that results in bivariate normal density forecasts. In experiments with 48-hour ahead forecasts of surface wind vectors over the Pacific Northwest, based on the University of Washington Mesoscale Ensemble (UWME), the postprocessed EMOS density forecast proved to bias correct and calibrate the raw ensemble forecast, therefore resulting in strongly improved deterministic and probabilistic predictive skill. When compared to the raw ensemble forecast and aggregated over calendar year 2008 and the Pacific Northwest, the Local EMOS technique reduced the bivariate mean absolute error by $13 \%$ and the bivariate mean energy score by $24 \%$.

There are several directions into which our bivariate EMOS method could be developed. In phases two and three of the estimation scheme, the exponential forgetting approach as in Pinson (2011) could be implemented, where the parameter estimates are updated in a computationally efficient, adaptive way. Furthermore, a geostatistical approach such as that of Kleiber et al. (2011) in the context of Bayesian model averaging (Raftery et al., 2005) could be developed in order to spread the Local EMOS estimates, which currently are available at observation locations only, over the model grid. Alternatively, if an analysis is used to fit the EMOS model, as was done by Pinson (2011), training data are available on the analysis grid, thereby allowing for a gridded Local EMOS approach.

A closer look at the scatterplots in Figure 3 suggests that the conditional distribution of the observed wind vector, given the ensemble forecast, tends to be skewed. Thus, our bivariate EMOS approach, which currently is based on bivariate normal densities, could be extended to allow for skewed distributions, such as bivariate skew-normal or skew- $t$ densities, similar to the wind vector time series methods proposed by Hering and Genton (2010). However, any such approach would be considerably more complex, and as it is more difficult to estimate a more complex predictive model, it is not clear whether or not such an extension would result in improved forecast performance.

Sloughter (2009) and Sloughter et al. (2011) proposed a bivariate version of the Bayesian model averaging technique (BMA; Raftery et al., 2005) for postprocessing ensemble forecasts of wind vectors. Like our EMOS method, the BMA approach results in a bivariate forecast density. However, the BMA forecast density is a finite mixture of bivariate, power-transformed normal densities and thus can be multimodal, as opposed to the EMOS forecast density, which is necessarily 
unimodal and elliptically symmetric. Thus, the basic setting of the EMOS approach is more parsimonious, thereby allowing for the key innovation in our work, namely the explicit modeling of the correlation between the $u$ and $v$ wind vector components, conditionally on the direction of the ensemble mean vector.

In contrast to the BMA and EMOS methods, the ensemble copula coupling technique (ECC; Schefzik 2011) and the postprocessing approach proposed by Pinson (2011) generate discrete forecast ensembles that are constrained to have the same number of members, and the same bivariate Spearman rank correlation coefficients, as the raw ensemble. Therefore, these methods are particularly well adapted to large ensembles, where there is no pronounced need for the transition from a discrete forecast ensemble to a density forecast, nor any need for statistical correction of the conditional correlation structure between the wind vector components. Accordingly, both Pinson (2011) and Schefzik (2011) tailored their methods to the 50-member European Centre for Mediumrange Weather Forecasts (ECMWF) ensemble, while our work considered the much smaller eightmember UWME, where the wind vector ensemble forecasts for any given location and valid time may show unreliable, physically unrealistic empirical correlation coefficients. This effect is caused by the small ensemble size and corroborates the need for a parametric correlation model.

In this paper, we considered probabilistic forecasts of wind vectors for a single location and valid time. While we modeled the bivariate correlation structure between the wind vector components, we considered the postprocessed bivariate density forecasts at each site individually, without modeling the dependence structure between locations. To address the latter, the methods developed in our paper could be combined with the spatial statistical techniques introduced by Gel et al. (2004). A common advantage of the method proposed by Pinson (2011) and the ECC technique (Schefzik, 2011) lies in their immediate extension to the calibration of spatio-temporal trajectories, in that the raw ensemble's bivariate rank dependence structure is inherited by the postprocessed ensemble, subject to the aforementioned caveats.

\section{Appendix: Verification methods}

In verifying probabilistic forecasts of a multivariate weather quantity, we use techniques introduced, studied and used by Gneiting et al. (2008), Gneiting (2011) and Pinson and Hagedorn (2011).

To assess the calibration of probabilistic forecasts of wind vectors, we use the multivariate rank histogram, which is a natural, direct generalization of the verification rank histogram or Talagrand diagram for a univariate quantity (Anderson, 1996; Hamill and Colucci, 1997; Talagrand et al, 1997) and can be interpreted analogously, in ways described by Hamill (2001). In particular, Ushaped multivariate rank histograms correspond to underdispersed ensembles, while inverse U- or hump-shaped histograms indicate overdispersed ensembles. For a calibrated ensemble, we expect a uniform rank histogram. For an ensemble with $m$ members, the multivariate verification rank is a possibly randomized number between 1 and $m+1$, and we refer to Gneiting et al. (2008) for the technical details in its construction. To quantify the departure of the rank histogram from unifor-

mity, we use the discrepancy or reliability index $\Delta$ proposed by Delle Monache et al. (2006), given by

$$
\Delta=\sum_{i=1}^{m+1}\left|f_{i}-\frac{1}{m+1}\right|,
$$


where $f_{i}$ is the observed relative frequency of verification rank $i=1, \ldots, m+1$. The UWME raw ensemble and ensemble copula coupling (ECC) techniques result in a discrete forecast ensemble with $m=8$ members, from which the construction of the multivariate rank histogram is straightforward. The Error Dressing techniques provide discrete forecast ensembles with $m=35$ members, and we bin the corresponding 36 ranks in the multivariate rank histogram into nine groups, comprising ranks $1-4, \ldots, 33-36$, respectively, to facilitate the comparison. For the Independent EMOS and bivariate EMOS techniques, we draw a simple random sample of size $m=8$ from the bivariate normal predictive distribution, and then compute the multivariate rank histogram.

To assess the overall quality of probabilistic forecasts, considering both calibration and sharpness, we use proper scoring rules (Gneiting and Raftery, 2007; Wilks, 2011). For a univariate weather quantity, such as wind speed, the proper continuous ranked probability score is defined as

$$
\operatorname{crps}(P, y)=\int_{-\infty}^{\infty}(P(x)-\mathrm{I}(x \geq y))^{2} \mathrm{~d} x=\mathbb{E}_{P}|X-y|-\frac{1}{2} \mathbb{E}_{P}\left|X-X^{\prime}\right|,
$$

where $P$ is the predictive distribution, here taking the form of a cumulative distribution function, $X$ and $X^{\prime}$ are independent random variables with cumulative distribution function $P$, and $y$ is the verifying value (Gneiting and Raftery, 2007). The term $\mathrm{I}(x \geq y)$ denotes an indicator function, equal to 1 if $x \geq y$, and equal to 0 otherwise, and $\mathbb{E}$ is the expectation operator. The absolute error is defined as

$$
\operatorname{ae}(P, y)=\left|\operatorname{med}_{P}-y\right|,
$$

where $\operatorname{med}_{P}$ is a median of the probability distribution $P$ Gneiting, 2011; Pinson and Hagedorn, 2011).

The energy score was introduced by Gneiting and Raftery (2007) and Gneiting et al. (2008) as a direct generalization of the continuous ranked probability score (7) in the evaluation of probabilistic forecasts of multivariate quantities. As we are interested in wind vectors, we restrict the discussion to bivariate weather quantities. The energy score then is defined as

$$
\operatorname{es}(P, y)=\mathbb{E}_{P}\|X-y\|-\frac{1}{2} \mathbb{E}_{P}\left\|X-X^{\prime}\right\|
$$

where $\|\cdot\|$ denotes the Euclidean norm in $\mathbb{R}^{2}, P$ is the predictive distribution, $X$ and $X^{\prime}$ are independent random vectors with distribution $P$, and $y \in \mathbb{R}^{2}$ is the verifying wind vector. For an ensemble forecast, the predictive distribution $P_{\text {ens }}$ has point mass $\frac{1}{m}$ at the member forecasts $x_{1}, \ldots, x_{m} \in \mathbb{R}^{2}$, and the energy score can be evaluated as

$$
\mathrm{es}\left(P_{\mathrm{ens}}, y\right)=\frac{1}{m} \sum_{j=1}^{m}\left\|x_{i}-y\right\|-\frac{1}{2 m^{2}} \sum_{i=1}^{m} \sum_{j=1}^{m}\left\|x_{i}-x_{j}\right\|
$$

We use this formula to compute the energy score for the UWME raw ensemble, Ensemble Copula Coupling (ECC) and Error Dressing techniques. For the Independent EMOS and bivariate EMOS techniques, we draw a simple random sample $x_{1}, \ldots, x_{k} \in \mathbb{R}^{2}$ from the corresponding predictive density, and replace the exact energy score (9) by the computationally efficient approximation

$$
\widehat{\mathrm{es}}(P, y)=\frac{1}{k} \sum_{i=1}^{k}\left\|x_{i}-y\right\|-\frac{1}{2(k-1)} \sum_{i=1}^{k-1}\left\|x_{i}-x_{i+1}\right\|
$$


where we use a sample of size $k=10,000$. Similar approximations apply to the continuous ranked probability score (7).

The natural generalization of the absolute error (8) is the bivariate absolute error

$$
\operatorname{bae}(P, y)=\left\|\operatorname{bmed}_{P}-y\right\|,
$$

where $\operatorname{bmed}_{P}$ denotes the bivariate or spatial median of the probability distribution $P$, defined as

$$
\operatorname{bmed}_{P}=\arg \min { }_{x \in \mathbb{R}^{2}} \mathbb{E}_{P}\|x-X\|,
$$

where $X$ is a random vector with distribution $P$ (Vardi and Zhang, 2000; Gneiting, 2011). For an elliptically symmetric distribution, such as a bivariate normal distribution, the bivariate median and the mean vector coincide. For other types of bivariate distributions, such as an ensemble forecast with point mass $\frac{1}{m}$ at the member forecasts $x_{1}, \ldots, x_{m} \in \mathbb{R}^{2}$, the bivariate median generally is different from the corresponding mean vector, and typically it needs to be determined numerically. For doing this we use the algorithm described by Vardi and Zhang (2000) and implemented in the $\mathrm{R}$ package ICSNP.

In practice, forecasting methods are assessed by averaging scores over a test period, resulting in the mean continuous ranked probability score (CRPS), mean absolute error (MAE), mean energy score (ES) and bivariate mean absolute error (bMAE), respectively. All these quantities are negatively oriented, that is, the smaller the better, and we report their values in the unit of meters per second.

\section{Acknowledgements}

The authors are grateful to Jeff Baars, Tom M. Hamill, Clifford F. Mass, Adrian E. Raftery and J. McLean Sloughter for discussions and providing data. Tom Hamill communicated to us the idea that underlies ensemble copula coupling (ECC), well before we noticed the independent discussions in the work of Bremnes (2007) and Krzysztofowicz and Toth (2008) and the term ECC was coined in Schefzik (2011).

\section{References}

Anderson, J. L. (1996). A method for producing and evaluating probabilistic forecasts from ensemble model integrations. Journal of Climate 9, 1518-1530.

Baars, J. (2005). Observations QC documentation. Available at http://www.atmos.washington.edu/mm5rt/qc_obs/qc_doc.html.

Bao, L., T. Gneiting, E. P. Grimit, P. Guttorp, and A. E. Raftery (2010). Bias correction and Bayesian model averaging for ensemble forecasts of surface wind direction. Monthly Weather Review 138, 1811-1821.

Bremnes, J. B. (2007). Improved calibration of precipitation forecasts using ensemble techniques. Part 2: Statistical calibration methods. Norwegian Meteorological Institute, Technical Report no. 04/2007. Available at http://met.no/Forskning/Publi kasjoner/Publikasjoner_2007/filestore/report04_2007.pdf. 
Delle Monache, L., J. P. Hacker, Y. Zhou, X. Deng, and R. B. Stull (2006). Probabilistic aspects of meteorological and ozone regional ensemble forecasts. Journal of Geophysical Research 111, D24307.

Eckel, F. A. and C. F. Mass (2005). Aspects of effective mesoscale, short-range ensemble forecasting. Weather and Forecasting 20, 328-350.

Gel, Y., A. E. Raftery, and T. Gneiting (2004). Calibrated probabilistic mesoscale weather field forecasting: The geostatistical output perturbation (GOP) method (with discussion). Journal of the American Statistical Association 99, 575-587.

Gneiting, T. (2011). Making and evaluating point forecasts. Journal of the American Statistical Association 106, 746-762.

Gneiting, T., F. Balabdaoui, and A. E. Raftery (2007). Probabilistic forecasts, calibration and sharpness. Journal of the Royal Statistical Society Series B 69, 243-268.

Gneiting, T. and A. E. Raftery (2007). Strictly proper scoring rules, prediction, and estimation. Journal of the American Statistical Association 102, 359-378.

Gneiting, T., A. E. Raftery, A. H. Westveld, and T. Goldman (2005). Calibrated probabilistic forecasting using ensemble model output statistics and minimum CRPS estimation. Monthly Weather Review 133, 1098-1118.

Gneiting, T., L. I. Stanberry, E. P. Grimit, L. Held, and N. A. Johnson (2008). Assessing probabilistic forecasts of multivariate quantities, with an application to ensemble predictions of surface winds. Test 17, 211-235.

Grimit, E. and C. Mass (2002). Initial results of a mesoscale short-range ensemble forecasting system over the Pacific Northwest. Weather and Forecasting 17, 192-205.

Hagedorn, R., T. M. Hamill, and J. S. Whitaker (2008). Probabilistic forecast calibration using ECMWF and GFS ensemble reforecasts. Part I: Temperature. Monthly Weather Review 136, 2608-2619.

Hamill, T. M. (2001). Interpretation of rank histograms for verifying ensemble forecasts. Monthly Weather Review 129, 550-560.

Hamill, T. M. and S. J. Colucci (1997). Verification of Eta-RSM short-range ensemble forecasts. Monthly Weather Review 125, 1312-1327.

Hering, A. and M. G. Genton (2010). Powering up with space-time wind forecasting. Journal of the American Statistical Association 105, 92-104.

Kann, A., C. Wittmann, Y. Wang, and X. Ma (2009). Calibrating 2-m temperature of limited area ensemble forecasts using high-resolution analysis. Monthly Weather Review 137, 3373-3387.

Kleiber, W., A. E. Raftery, J. Baars, T. Gneiting, C. F. Mass, and E. Grimit (2011). Locally calibrated probabilistic temperature forecasting using geostatistical model averaging and local Bayesian model averaging. Monthly Weather Review 139, 2630-2649. 
Krzysztofowicz, R. and Z. Toth (2008). Bayesian processor of ensemble (BPE): Concept and implementation. Workshop slides 4th NCEP/NWS Ensemble User Workshop, Laurel, Maryland. Available at http://www.emc.ncep.noaa.gov/gmb/ens/ens2008/Krzysztofowicz_Pres entation_Web.pdf.

Leutbecher, M. and T. N. Palmer (2008). Ensemble forecasting. Journal of Computational Physics 227, 3515-3539.

Marquis, M., J. Wilczak, M. Ahlstrom, J. Sharp, A. Stern, C. J. Smith, and S. Calvert (2011). Forecasting the wind to reach significant penetration levels of wind energy. Bulletin of the American Meteorological Society 92, 1159-1171.

National Weather Service (1998). Automated Surface Observing System (ASOS) User's Guide. Available at http://www.weather.gov/asos/aum-toc.pdf.

Pinson, P. (2011). Adaptive calibration of $(u, v)$-wind ensemble forecasts. Quarterly Journal of the Royal Meteorological Society, in press.

Pinson, P. and R. Hagedorn (2011). Verification of the ECMWF ensemble forecasts of wind speed against analyses and observations. Meteorological Applications, in press.

R Development Core Team (2011). R: A Language and Environment for Statistical Computing. Vienna, Austria: R Foundation for Statistical Computing. ISBN 3-900051-07-0.

Raftery, A. E., T. Gneiting, F. Balabdaoui, and M. Polakowski (2005). Using Bayesian model averaging to calibrate forecast ensembles. Monthly Weather Review 133, 1155-1174.

Roulston, M. and L. Smith (2003). Combining dynamical and statistical ensembles. Tellus A 55, $16-30$.

Schefzik, R. (2011). Ensemble Copula Coupling. Diploma thesis, Faculty of Mathematics and Informatics, University of Heidelberg.

Schölzel, C. and P. Friederichs (2008). Multivariate non-normally distributed random variables in climate research - Introduction to copulas. Nonlinear Processes in Geosciences 15, 761-772.

Schuhen, N. (2011). Ensemble Model Output Statistics for Wind Vectors. Diploma thesis, Faculty of Mathematics and Informatics, University of Heidelberg.

Sloughter, J. M. (2009). Probabilistic Weather Forecasting Using Bayesian Model Averaging. Ph.D. thesis, Department of Statistics, University of Washington.

Sloughter, J. M., T. Gneiting, and A. E. Raftery (2010). Probabilistic wind speed forecasting using ensembles and Bayesian model averaging. Journal of the American Statistical Association 105, $25-35$.

Sloughter, J. M., T. Gneiting, and A. E. Raftery (2011). Probabilistic wind vector forecasting using ensembles and Bayesian model averaging. Monthly Weather Review, submitted. 
Talagrand, O., R. Vautard, and B. Strauss (1997). Evaluation of probabilistic prediction systems. In Proc. Workshop on Predictability, pp. 1-25. Reading, UK, European Centre for Medium-Range Weather Forecasts.

Thorarinsdottir, T. L. and T. Gneiting (2010). Probabilistic forecasts of wind speed: Ensemble model output statistics by using heteroscedastic censored regression. Journal of the Royal Statistical Society Series A 173, 371-388.

Thorarinsdottir, T. L. and M. S. Johnson (2011). Probabilistic wind gust forecasting using nonhomogeneous Gaussian regression. Monthly Weather Review, in press.

Vardi, Y. and C.-H. Zhang (2000). The multivariate $L_{1}$-median and associated data depth. Proceedings of the National Academy of Sciences of the United States of America 97, 1423-1426.

Whitaker, J. S. and A. F. Loughe (1998). The relationship between ensemble spread and ensemble mean skill. Monthly Weather Review 126, 3292-3302.

Wilks, D. S. (2009). Extending logistic regression to provide full-probability-distribution MOS forecasts. Meteorological Applications 16, 361-368.

Wilks, D. S. (2011). Statistical Methods in the Atmospheric Sciences (third ed.). Academic Press.

Wilks, D. S. and T. M. Hamill (2007). Comparison of ensemble-MOS methods using GFS reforecasts. Monthly Weather Review 135, 2379-2390. 